\title{
A Metodologia da Problematização (MP) como estratégia de integração ensino-serviço em cursos de graduação na área da saúde
}

\section{Problematization Methodology (PM) as an integration strategy between teaching and service in health undergraduate courses}

\author{
Marta Neves Campanelli Marçal Vieira ${ }^{1}$, Maria Paula Panúncio-Pinto²
}

\begin{abstract}
RESUMO
A formação de profissionais para atuar na área da saúde deve superar o modelo tradicional de educação centrado no paradigma cartesiano/flexneriano, fixado na fragmentação e especialização do conhecimento. A graduação em saúde precisa alcançar estratégias de ensino-aprendizagem que possibilitem a construção coletiva dos conhecimentos, a permanente integração teoria-prática referenciada na realidade social concreta. Tomando a perspectiva das metodologias ativas de ensino-aprendizagem, o objetivo deste artigo é apresentar e discutir a Metodologia da Problematização (MP) como estratégia políticopedagógica de aproximação à realidade e construção da autonomia na formação de profissionais da saúde. Além disso, apresenta as diferenças entre a MP a utilização de outras formas de problematização e solução de problemas como estratégia de ensino. Finalmente discute os limites e possibilidades do método.
\end{abstract}

Palavras-chave: Ensino Superior, Aprendizagem Ativa. Metodologia da Problematização. Saúde /Educação Superior.

\begin{abstract}
Health professionals training should overcome the traditional education model centered on the Cartesian /Flexnerian paradigm, established on specialized and fragmented knowledge and on methodologies for knowledge transfer and deposit. Undergraduate in health courses needs to achieve teaching and learning strategies that allow the collective construction of knowledge and permanent integration between theory
\end{abstract}

1. Professora Doutora do Departamento de Puericultura e Pediatria da Faculdade de Medicina de Ribeirão Preto da Universidade de São Paulo.

2. Professora Doutora do Departamento de Neurociências e Ciências do Comportamento da Faculdade de Medicina de Ribeirão Preto da Universidade de São Paulo.
Correspondência:

Profa. Dra. Maria Paula Panúncio-Pinto Departamento de Neurociências e Ciências do Comportamento Hospital das Clínicas da FMRP- Campus da USP CEP: 14048-900 - Ribeirão Preto mapaula@fmrp.usp.br 
and practice based on social reality. Taking the perspective of active methods of teaching and learning, the aim of this paper is to present and discuss the Problematization Methodology (PM) as a political pedagogical strategy of approach to reality and building autonomy in health professionals training. Moreover, it presents the differences between the PM and the use other forms of problematization and problem solving as a teaching strategy. Finally discusses the limits and possibilities of the method.

Keywords: Higher Education. Problem-Based Learning. Problematization Methodoly. Health / Education, Higher.

\section{Principais pontos de interesse}

1. A formação de profissionais para atuar na área da saúde demanda a superação do modelo tradicional de educação centrado no paradigma cartesiano/ flexneriano, fixado na fragmentação e especialização do conhecimento.

2. As chamadas metodologias ativas de ensino e aprendizagem constituem-se como estratégia para ultrapassar objetivos que se restringem ao domínio técnico-científico e baseiam-se no princípio teórico da autonomia, e no pressuposto de um estudante capaz de autogerenciar seu processo de formação.

3. A Metodologia da Problematização (MP) e outras formas de utilizar problemas como estratégia de ensino-aprendizagem na educação superior, são apontadas como alternativa para introduzir modelos inovadores pois quando o estudante interage com a cultura sistematizada de forma ativa, como ator do processo de construção do conhecimento, ocorre a aprendizagem significativa.

4. O esquema de progressão pedagógica que estrutura a aplicação do método apresenta o formato de um arco, e percorre etapas que envolvem observação, identificação de problemas, reflexão, teorização, hipótese de solução e propostas, e o alvo é sempre a realidade e as possibilidades de transformá-la, através da solução do problema colocado.

5. "Problematização", "Metodologia da Problematização" (MP) e "Aprendizagem Baseada em Resolução de Problemas" (ABRP), tradução mais usual para Problem Based Learning (PBL) não são sinônimos em termos de metodologia de ensino. O PBL e a MP são métodos específicos, com estrutura e procedimentos próprios, e as adaptações que eventualmente são feitas ao utilizar problemas em sala de aula não correspondem necessariamente a um ou a outro método.

\section{Considerações Iniciais}

No contexto da educação universitária "aprender" ganha um novo significado, uma vez que o estudante escolheu uma carreira e ao deixar a Universidade será um profissional, com compromissos assumidos frente à sociedade. ${ }^{1}$

Nessa perspectiva aumenta a responsabilidade do estudante com sua formação e são esperados envolvimento, iniciativa, responsabilidade e autonomia. O professor passa a ocupar o lugar de mediador na aquisição e atribuição de sentido ao conhecimento diante de realidades sociais concretas ${ }^{2}$, uma vez que a apreensão do sentido da expressão "aprendizagem significativa" aponta para a superação de objetivos educacionais baseados exclusivamente na lógica e na técnica. Especificamente em relação à formação de profissionais para atuar na área da saúde, tem se discutido a necessidade de superar o modelo tradicional de educação centrado no paradigma cartesiano/ flexneriano, fixado na fragmentação e especialização e em metodologias de transferência e depósito de conhecimento. A graduação em saúde precisa ultrapassar objetivos que se restringem ao domínio técnicocientífico, alcançando estratégias de ensino-aprendizagem que possibilitem a construção coletiva dos conhecimentos e a permanente integração teoria-prática referenciada na realidade social concreta. Isso implica em romper com a postura de transmissão de informações, na qual estudantes são considerados como sujeitos passivos cuja única preocupação seria recuperar informações quando solicitados. ${ }^{3}$

Outros aspectos do modelo tradicional de educação são questionados na construção da aprendizagem significativa tais como a concepção de conhecimento "indiscutível, pronto e acabado"; a memorização como operação essencial do processo de aprendizagem e a transmissão de conteúdo em aulas expositivas. $^{4}$ 
As chamadas metodologias ativas de ensino e aprendizagem emergem como estratégia de transformação. As metodologias ativas se baseiam no princípio teórico da autonomia, e no pressuposto de um estudante capaz de autogerenciar seu processo de formação. ${ }^{5}$ Igualmente, derivam dos pilares da educação para o século XXI ${ }^{6}$ dentre os quais o "o aprender a aprender" se destaca como constituinte da autonomia.

Nesse contexto, a Metodologia da Problematização (MP) e outras formas de utilizar problemas como estratégia de ensino-aprendizagem na educação superior, são apontadas como alternativa para introduzir modelos inovadores.

Quando o estudante interage com a cultura sistematizada de forma ativa, como ator do processo de construção do conhecimento, ocorre a aprendizagem significativa. Na observação ou reflexão sobre a realidade, em cenários de práticas ou em sala de aula, são obtidos problemas que serão estudados, criando desafios cognitivos constantes para professores e estudantes. Para estimular os estudantes, os conhecimentos prévios são considerados para dar continuidade ao processo de aprendizagem, através de situações-problema outras necessidades são provocadas, analisadas criticamente resultando na reelaboração de associações e de novos conhecimentos e sentidos. ${ }^{3,5}$

Antes de apresentar e discutir possibilidades constituição de pequenos grupos, a presença do tutor, a substituição das aulas por "sessões" ou tutorias. Também em relação à MP, é necessário que a organização curricular favoreça sua adoção como metodologia de ensino, adotada parcial ou totalmente no cotidiano do processo ensino-aprendizagem, mas vez que prevê a saída da sala de aula para o contexto da realidade concreta.

Neste artigo serão apresentados os componentes da Metodologia da Problematização, as possibilidades e os requisitos para sua utilização em cursos de graduação, como estratégia de ensino-aprendizagem.

\section{Metodologia da Problematização}

A Metodologia da Problematização (MP) foi proposta, inicialmente, por Bordenave e Pereira e seu ponto de partida é o pensamento freireano, uma vez que leva em conta a realidade do sujeito (estudante), sua experiência e conhecimentos prévios. ${ }^{8}$

A MP utiliza-se de um esquema elaborado por Charles Maguerez denominado "Método do Arco". 9 $\mathrm{O}$ esquema de progressão pedagógica que estrutura a aplicação do método apresenta o formato de um arco, e sofreu algumas adaptações em forma e conteúdo em relação ao original proposto por Maguerez (Figura 1).., 10 de utilização de situações- problema como estratégia de ensino-aprendizagem é necessário esclarecer que

"Problematização", "Metodologia da Problematização" e "Aprendizagem Baseada em Resolução de Problemas" (ABRP), tradução mais usual para Problem Based Learning (PBL) ${ }^{7}$ não são sinônimos, em termos de metodologia de ensino.

O PBL e a MP são métodos específicos, com estrutura e procedimento próprios, e as adaptações que eventualmente são feitas ao utilizar problemas em sala de aula não correspondem necessariamente a um ou outro método. Muitas vezes os termos PBL e MP são utilizados de forma equivocada. Especificamente em relação ao PBLé necessário considerar que sua aplicação depende de toda uma organização curricular que deve criar condições para que o método aconteça no conjunto dos componentes curriculares, como a

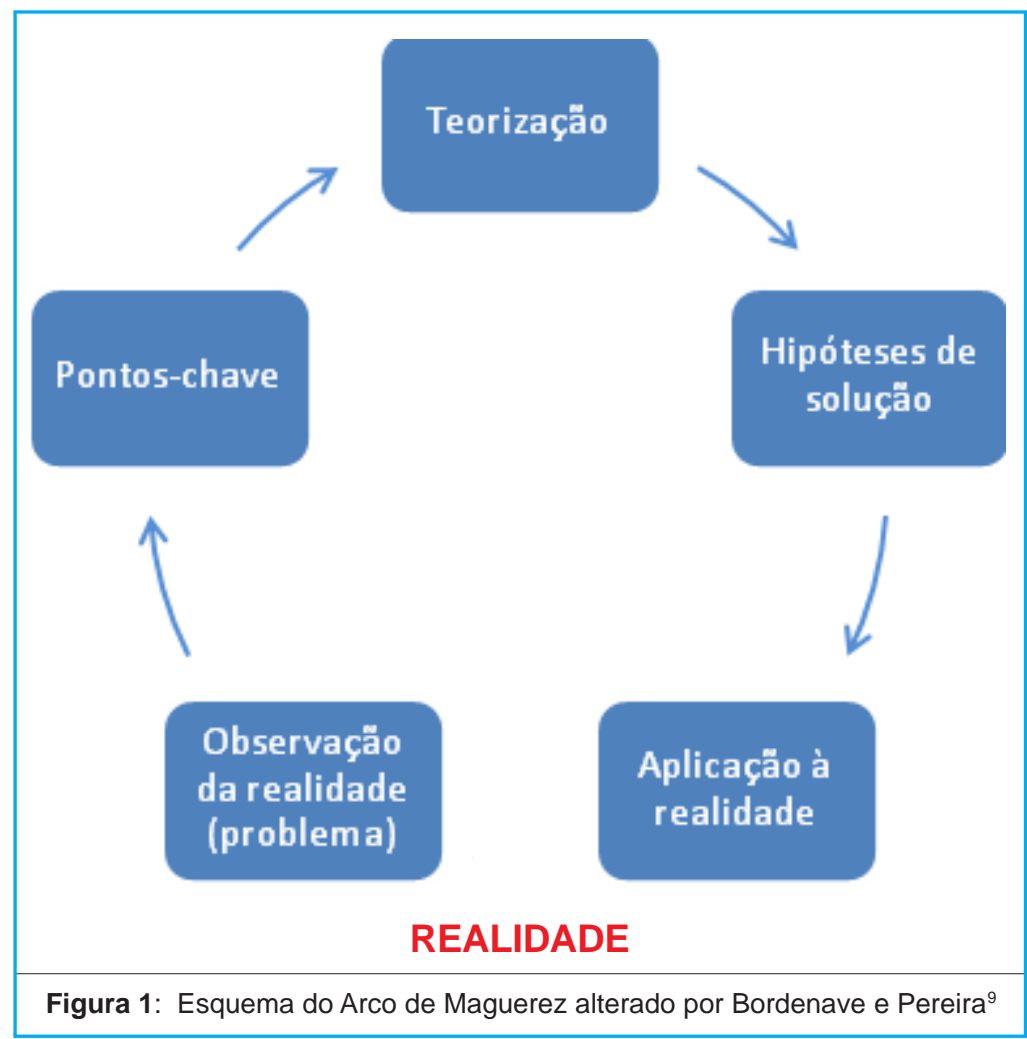


A estrutura do Método do Arco parte da realidade, e percorre etapas que envolvem observação, identificação de problemas, reflexão, teorização, hipótese de solução e propostas. O alvo é sempre a realidade e as possibilidades de transformá-la, através da solução do problema colocado. Para tanto, o Esquema do Arco de Maguerez se desenvolve em cinco etapas ${ }^{11}$ :

1. Observação da realidade concreta: estudantes são orientados a observar determinada realidade a partir de um tema ou conteúdo, registrando sistematicamente suas percepções com foco no tema. Devem problematizar dificuldades, falhas, contradições, discrepâncias e conflitos. Um ou mais problemas são escolhidos para estudo e investigação, podendo ocorrer a formação de pequenos grupos. O professor tem o papel de animador da discussão, auxiliando na formulação final e redação do problema que subsidiará as etapas seguintes.

2. Determinação de pontos-chave: os estudantes refletem sobre a gênese do problema, identificando a sua multideterminação e complexidade, questionando sua dimensão social e os possíveis determinantes que afetam o contexto no qual o problema ocorre e que não são evidenciados. Numa análise mais criteriosa do problema, buscam alternativas para sua solução. O professor orienta os estudantes na produção de uma nova síntese e seleção dos pontos mais relevantes (pontos-chave), que serão estudados na próxima etapa por meio de conhecimentos teóricos para compreender o problema com maior profundidade (tópicos ou perguntas).

3. Teorização: os estudantes se organizam para buscar conhecimentos e informações sobre o do problema em variadas fontes (pesquisa bibliográfica, leitura, entrevistas, consultas a especialistas). As informações são tratadas, organizadas e analisadas, discute-se sua validade e pertinência para a resolução do problema, verificando-se se as hipóteses foram confirmadas. $\mathrm{O}$ registro dessa etapa e das conclusões dela extraídas é necessário para o desenvolvimento da etapa seguinte.

4. Hipóteses de solução: a partir do estudo realizado são buscados os elementos para a elaboração de possíveis soluções, de forma criativa e crítica. $\mathrm{O}$ que precisa e pode ser feito para que o problema seja solucionado? As hipóteses são construídas após o estudo, como consequência da compreensão da realidade, investigando o problema de todos os ângulos possíveis.
5. Aplicação prática à realidade: de caráter prático, esta etapa completa a cadeia dialética ação - reflexão - ação, retornando ao ponto de partida, que é a realidade social. Ao adotar esta trajetória, os estudantes devem tomar decisões e executá-las, indo além do exercício intelectual, pois assumiram compromisso com a transformação do seu meio, mesmo que em pequena dimensão.

Para cada etapa do arco, define-se um conjunto de ações possíveis, as quais devem ser associadas à saberes específicos. Esse pensamento organiza e estrutura o método, a partir de um encadeamento concreto de ações (Quadro 1). ${ }^{12}$

Como é possível perceber, a MP tem como principais objetivos desencadear o pensamento reflexivo e mobilizar o potencial social e político do estudante. Outros objetivos são estimular o raciocínio e o desenvolvimento de habilidades intelectuais. ${ }^{13}$

O professor ou orientador que elege essa metodologia de ensino deixa de atuar como fonte central de informação ou de decisão das condutas e assume a condução metodológica do processo. A MP requer a dedicação de mais tempo para o ensino e para as atividades extramuros, exigindo a disponibilidade do professor para pesquisar, acompanhar e cooperar no aprendizado crítico do estudante, num processo compartilhado de construção do conhecimento. Tudo isso vai exigir do professor disponibilidade para lidar com situações imprevistas, habilidade para promover e participar do diálogo, paciência e segurança emocional para trabalhar com os estudantes, capacidade de sistematizar as informações e cuidado para dar um feedback imediato para a solução do problema. ${ }^{3,14}$

Além disso, nessa metodologia o professor não tem controle absoluto dos resultados em termos de conhecimentos e deve apresentar uma postura metodológica em relação ao processo de pesquisa que o estudante deverá realizar ativamente, e uma postura política coerente em relação ao processo educativo e aos problemas relativos ao tema em estudo.

Pelas características do trabalho com o conhecimento, após o estudo de um problema poderão surgir outros desdobramentos sobre o tema, exigindo dos envolvidos o contato com conteúdos que não foram previstos pelo professor, num primeiro momento, mas que precisarão ser investigados por serem relevantes à compreensão do problema.

Em todas as etapas do processo, os resultados não são previstos e podem ultrapassar os aspectos téc- 


\section{Quadro 1: Etapas do Método do Arco e ações correspondentes}

Etapas Ações

Etapa 1: Observação da realidade concreta

- Identificar o recorte de realidade a ser observado

- Eleger a forma de observação

- Preparar o necessário para a observação

- Realizar a observação (num formato definido ou possível)

- Registrar as observações

- Analisar o conteúdo do registro problematizando-o

- Eleger o foco do estudo (o problema) a partir de um critério

- Redigir o problema

- Justificar a escolha do problema

Etapa 2: Determinação de pontos-chave
- Refletir a respeito do problema

- Identificar possíveis fatores associados ao problema

- Identificar possíveis determinantes do problema

- Redigir a reflexão ( sentido para o estudo a partir das explicações da existência do problema)

- Analisar a reflexão, captando os vários aspectos envolvidos no problema

- Eleger aspectos que serão estudados na etapa seguinte

- Redigir os pontos-chave (síntese clara- perguntas ou tópicos)
- Eleger a forma de estudar cada ponto-chave

- Preparar os instrumentos de coleta de informação

- Testar os instrumentos

- Organizar as condições para a aplicação dos instrumentos

- Coletar as informações (aplicação dos procedimentos e instrumentos definidos)

- Tratar as informações

- Analisar e discutir as informações

- Estabelecer relações entre as diferentes informações

- Concluir em função do problema, verificando se as hipóteses explicativas iniciais foram confirmadas, negadas ou não foram consideradas na Teorização

- Registrar toda a Teorização
- Elaborar as hipóteses de solução para o problema,

- Abranger diferentes instâncias ou níveis de ação visando à transformação daquela parcela de realidade estudada

- Usar criatividade para encontrar ações novas

- Explicar/argumentra as hipóteses• elaboradas

- Registrar toda a elaboração
Etapa 5: Aplicação à realidade
- Analisar a aplicabilidade das hipóteses

- Eleger as possíveis de por em prática (exequibilidade, urgência, prioridade)

- Planejar a execução das ações pelas quais se compromete

- Executar as ações planejadas

- Registrar todo o processo, analisando os resultados 
nico-científicos, surpreendendo positivamente o professor e o grupo de estudantes. Mas, o professor pode identificar que o conteúdo apreendido pelos estudantes não corresponde às suas expectativas, o que demandará a necessidade de providenciar outra forma e momento para recuperar os aspectos do programa que não foram atingidos naquele tema. ${ }^{11,3}$

\section{Limites e possibilidades da utili- zação da Metodologia da Proble- matização}

Considerando que a MP se baseia, a exemplo do PBL, em um método específico, com etapas e ações definidas em termos do que se espera do professor e do estudante, a sua utilização deve necessariamente estar prevista pelo planejamento educacional da instituição de ensino, a qual integra o Projeto Político Pedagógico (PPP), o plano de aprendizagem e o plano de aula.

Nesse sentido, ainda que sua utilização não demande grandes mudanças em termos de estrutura física, é necessário que ela esteja prevista na organização curricular, para que os ajustes necessários sejam possíveis, bem como para que a utilização da metodologia esteja em consonância com os objetivos do curso e com o perfil final definido para o graduando.

São escolhas que se materializam a partir do PPP, com a definição dos cenários de ensino, a partir da integração dos eixos e componentes curriculares ou disciplinas, considerando a especificidade de cada etapa da formação. As definições realizadas a partir do PPP podem refletir a escolha por uma utilização seletiva da MP, em alguns componentes curriculares ou para alguns módulos de determinadas disciplinas.

Tomando como ponto de partida o planejamento educacional da instituição de ensino, é necessário considerar que a MP pode não ser apropriada para todos os conteúdos, para os quais podem ser indicadas outras alternativas metodológicas. Nesse sentido, destaca-se que quando os temas envolvem aspectos sociais, éticos, econômicos e políticos, a MP pode consistir num recurso de possibilidades interessantes para o aprendizado. ${ }^{11}$

A MP requer o trabalho em pequenos grupos, com eventual divisão de tarefas e fica,então à cargo do professor supervisionar os trabalhos para garantir sua integração. $\mathrm{O}$ trabalho em grupo contribui para o desenvolvimento de habilidades importantes para cooperar, conviver, comunicar-se, planejar. ${ }^{3}$
A problematização leva ao desenvolvimento de atividades que permitem ao estudante pensar para além de sua área de formação e especialidade, possibilitando múltiplas abordagens conceituais e o exercício da interdisciplinaridade.

Diante de tantas possibilidades e do potencial da MP é necessário considerar seus limites e os obstáculos para que todo seu potencial se concretize.

Há que se pensar no perigo de tomá-la como simples instrumento técnico, utilizado a partir de pequenas adaptações ao tradicional. SE a MP for utilizada de forma desvinculada ao $\mathrm{PPP}^{15}$ corre-se o risco da simples reprodução de experiências ${ }^{3}$ num pretenso modelo inovador, invocado em nome das demandas do mercado, centrado no desenvolvimento de competências voltadas para a realidade, mas que não problematiza o ensino e o papel do professor tampouco a necessidade de refletir e criticar, para transformar.

Também é necessário considerar a resistência à mudança, que pode vir tanto dos estudantes quanto dos professores, as pequenas adaptações ao tradicional que não se configuram como real transformação nas práticas de ensino, bem como a compreensão equivocada do método, fatores que alteram os resultados esperados. ${ }^{16}$

As dificuldades e limites apontam para a necessidade de investimento constante no desenvolvimento profissional do professor universitário, uma vez que deixar a perspectiva das tradicionais aulas expositivas exige formação e capacitação docente. Como toda a metodologia ativa, a MP desafia o professor a sair de sua zona de conforto, preparar-se didática e pedagogicamente para o exercício da docência, numa perspectiva investigativa e dinâmica. Nessa direção, o docente é chamado a reavaliar a dimensão política de suas práticas e a reconsiderar seu compromisso com o tripé ensino-pesquisa-assistência.

\section{Considerações Finais}

A adoção de metodologias ativas é um grande desafio na reformulação dos Projetos Políticos Pedagógicos, tarefa que todos os cursos da área da saúde estão enfrentando a partir das Diretrizes Curriculares Nacionais (DCNs), que nos últimos anos têm apontado para o compromisso com a realidade social concreta como a meta fundamental da formação na graduação.

De certa forma, demandas colocadas pelas DCNs decorrem de transformações na organização do campo da saúde brasileiro, que se intensificaram com 
a regulamentação do Sistema Único de Saúde (SUS) com a publicação da Lei 8080/90 ${ }^{17}$ : mudanças nas práticas de saúde resultaram no reconhecimento de necessárias alterações no processo de formação e desenvolvimento profissional.

Uma vez que a legislação define que os serviços públicos que integram o SUS devem ser o campo de prática para o ensino e a pesquisa, coloca-se o desafio de ampliar a interface Universidade-Comunidade, com a diversificação dos cenários de ensino, levando estudantes e professores a deslocarem-se da tradicional sala de aula, para equipamentos sociais no território.

A partir de 2002, ações conjuntas do entre o Ministério da Saúde e o Ministério da Educação resultaram na criação de programas que reforçam o deslocamento sala de aula $\Rightarrow$ comunidade, originando uma nova política de formação de recursos humanos para o SUS. Destacam-se o AprenderSUS, o Programa Nacional de Reorientação da Formação Profissional em Saúde - Pró- Saúde e o Programa de Orientação pelo Trabalho para a Saúde - Pet- Saúde. ${ }^{18-21}$

A integração entre as Instituições de Ensino Superior (IES) e o SUS, proposta pela legislação e materializada pelos programas citados, têm constituído mudanças na graduação, objetivando uma formação profissional que contemple o sistema de saúde vigente, privilegiando a prática social, de modo a articular organicamente ensino-serviço. ${ }^{22}$

Nesse sentido, é importante considerar que a aproximação à realidade concreta que a Metodologia da Problematização permite, responde a necessidade de preparar os futuros profissionais para o aprendizado contínuo, considerando demandas de uma sociedade em constante transformação. Particularmente, no caso das profissões da saúde, atende à demanda política de aproximação das IES ao SUS, garantindo a formação acadêmico-científica, ética e humanística para o desempenho profissional contextualizado socialmente.

A MP tem sido utilizada em cursos técnicos e de graduação da área da saúde e, de forma geral, os resultados são descritos como positivos. O potencial mais evidenciado do método é sua abertura para explorar temas relacionados diretamente com o social, ou a vida em sociedade. As possibilidades derivadas dessa perspectiva são especialmente importantes na formação dos profissionais que realizam prestação de serviços à comunidade, como é o caso das profissões da saúde. ${ }^{16,3}$

\section{Referências}

1. Severino AJ. Metodologia do trabalho científico. 23를 Ed. São Paulo: Cortez, 2007.

2. Panúncio-Pinto MP; Pfeifer LI. Metodologia do trabalho acadêmico- científico. Apostila didática. Ribeirão Preto: FMRP, 2009.

3. Cyrino EG; Toralles-Pereira ML.Trabalhando com estratégias de ensino- aprendizado por descoberta na área da saúde: a problematização e a aprendizagem baseada em problemas. Cad Saúde Pública. 2004; 20: 780-8.

4. Anastasiou LGC. Metodologia de ensino na universidade brasileira: elementos de uma trajetória. In: M.E. Castanho e S. Castanho (ORGs) Temas e Textos da Educação Superior. Capinas: Papirus, 2001.

5. Mitre SM; Siqueira-Batista R; Girardi-de-Mendonça JM; Morais-Pinto NM; Meirelles CAB; Pinto-Porto C; et al. Metodologias ativas de ensino-aprendizagem na formação profissional em saúde: debates atuais. Ciênc. saúde coletiva. 2008; 13(Sup 2):2133-44.

6. Morin E. Os sete saberes necessários à educação do futuro. 2aㅡ름õo. São Paulo: Cortez; Brasília,DF: UNESCO, 2000.

7. Leite L; Esteves E. Trabalho em grupo e aprendizagem baseada na resolução de problemas: um estudo com futuros professores de química e física. In: Actas International Conference PBL. Lima, Peru, 2006

8. Berbel NAN. A metodologia da problematização e os ensinamentos de Paulo Freire: uma relação mais que perfeita. In: Berbel NAN. Metodologia da problematização: fundamentos e aplicações. Londrina: UEL; 1999.

9. Berbel NAN; Gamboa SAS. A metodologia da problematização com o Arco de Maguerez: uma perspectiva teórica e epistemológica. Filosofia e Educação (Online), ISSN 19849605. 2012; 3: № 2.

10. Berbel NAN. A Metodologia da Problematização em três versões no contexto da didática e da formação de professores. Rev Diálogo Educ.. 2012; 12: 103-20.

11. Berbel NAN. A problematização e a aprendizagem baseada em roblemas: diferentes termos ou diferentes caminhos? Interface comun. saúde educ., 1998; 2: 139-54.

12. Colombo AA; Berbel NAN. A Metodologia da Problematização com o Arco de Maguerez e sua relação com os saberes de professores. Semina: Ciências Sociais e Humanas. 2007; 28: $121-46$.

13. Berbel NAN. Metodologia da Problematização: uma alternativa metodológica apropriada para o Ensino Superior. Semina: Ciências Sociais e Humanas. 1995; Ed. Especial 16: 9-19.

14. Freitas RAMM. Ensino por problemas: uma abordagem para o desenvolvimento do aluno. Educação e Pesquisa, São Paulo. 2012 ; 38: 403-18.

15. Batista N; Batista SH; Goldenberg P; Seiffert O; Sonzogno MC. O enfoque problematizador na formação de profissionais da saúde. Rev Saúde Pública. 2005; 39: 231-7.

16. Azevedo JA. Fundamentos filosóficos da pedagogia de Paulo Freire. Akrópolis Umuarama. 2010; 18: 37-47.

17. Brasil. Lei oㅡ 8080, de 19 de setembro de 1990. Dispõe sobre as condições para a promoção, proteção e recuperação da saúde, a organização e o funcionamento dos serviços correspondentes e dá outras providências. Diário Oficial da União. Brasília, 20 set. 1990; Seção 1. 
18. Brasil. Ministério da Educação. Ministério da Saúde. AprenderSUS: o SUS e os cursos de graduação na área da saúde.Série B: Textos Básicos de Saúde.Brasília, DF. 2004. Disponível em http://bvsms.saude.gov.br/bvs/publicacoes/ cartilha_aprender_sus.pdf acesso em 13/10/2014.

19. Brasil. Ministério da Saúde. Ministério da Educação. Programa Nacional de Reorientação da Formação Profissional em Saúde - Pró-Saúde: objetivos, implementação e desenvolvimento potencial. Brasília: Ministério da Saúde, 2007

20. Brasil. Ministério da Saúde, Ministério da Educação. Pró-Saúde: Programa Nacional de Reorientação da Formação Profissional em Saúde. Brasília: Ministério da Saúde, 2005.
21. Brasil. Ministério da Saúde. Portaria Interministerial ํㅜ 1.802, de 26 de agosto de 2008. Institui o Programa de Educação pelo Trabalho para a Saúde - PET-Saúde. Diário Oficial da União, Brasília, 26 ago. 2008. Seção 1, p.27

22. Hora DL, Erthal RMC, Souza CTV, Hora EL. Propostas inovadoras na formação do profissional para o Sistema Único de Saúde. Trab educ saúde (Rio de Janeiro). 2013; 11: 471-86. 